\title{
Detection of Time Series Change Point of Stock Yield Based on Bayesian Method
}

\author{
YING Yi rong, YING Feng jie and LI Su zhen \\ College of Economics, Shanghai University, shanghai, 200444, China
}

\section{Keywords: Bayesian Method; Mean Change Point; Posterior Probability Ratio}

Abstract. In this paper we suggest using the method of posterior probability to extend the ICSS algorithm based on the formula of posterior probability ratio. The empirical results of Shanghai Composite Index show that the posterior probability algorithm is convenient and effective.

\section{Model Analysis}

Time series will have critical sudden change in the sense of statistics with social change and development; therefore, the structural change is always the frontier of the statistical research, especially the detection of change point of financial time series has been concerned. As one of the typical financial time series, the stock price can be considered as an integral part in research on detection of change point.

Abraham and Box(1979) ${ }^{[1]}$ put forward the Bayesian Method of identifying abnormal point in time series. Inclan (1993) ${ }^{[2]}$ studied the variance multiple change point of the yield by the Bayesian Method for the first time, and validated the effectiveness of the model with the famous IBM stock price time series as the example. However, the model has disadvantage of large calculation amount, difficult to apply to the longer time series.

Consider a time series of yield obeying normal distribution $\left\{r_{t}\right\}, t=1,2, \ldots, T$. Assume there are $\mathrm{M}$ change points in the time series, the subscripts of change points $\kappa=\left(\kappa_{1}, \kappa_{2}, \ldots, \kappa_{M}\right)^{\prime}$, $1 \leq \kappa_{1}<\kappa_{2}<\ldots<\kappa_{M}<T$, and $M=1, \ldots, T-1$. In order to facilitate the mathematical expression, appoint $\kappa_{0}=0$ and $\kappa_{M+1}=T$. The change points divide the time series into $M+1$ time periods, and mean of yield distribution in each time period is same. The variance of distribution is different $\tau_{t}^{2}, t=1,2, \ldots, T$.

$$
r_{t} \sim \mathrm{N}\left(\theta_{\mathrm{j}}, \tau_{t}^{2}\right), \kappa_{\mathrm{j}}<t \leq \kappa_{j+1}, j=0,1, \ldots, M .
$$

In the time period $\mathrm{j}$, the mean of yield distribution is $\theta_{j}$, and the observation number is $d_{j}=\kappa_{j+1}-\kappa_{j}, j=0,1, \ldots, M$. The current problem is how to use yield sample $r=\left(r_{1}, r_{2}, \ldots, r_{T}\right)^{\prime}$ to estimate the position of change point $\kappa=\left(\kappa_{1}, \kappa_{2}, \ldots, \kappa_{M}\right)^{\prime}$.

Inclan $(1993)^{[2]}$ gave the joint distribution density of the entire yield time series based on the hypothesis that mean and variance of yield distribution in each time period were same

$$
p(r \mid \tau, \kappa, M)=(2 \pi)^{-T / 2} \prod_{j=0}^{M} \tau_{j}^{-d_{j}} \exp \left\{-\frac{1}{2 \tau_{j}^{2}} \sum_{t=\kappa_{j}+1}^{\kappa_{j+1}} r_{t}^{2}\right\} .
$$

In the time period $\mathrm{j}$, mean of yield distribution is $\theta_{j}$ and variance is $\tau_{j}^{2}$. It assumes that mean of yield distribution in each time period is zero; therefore, there is no mean parameter in the expression of joint distribution density.

Changes are made based on Formula (2). First, notice that the number of change points is actual the dimension of vector at the positions of change points, and the function $M=N(\kappa)$ can be used for expression. Second, abandon the hypothesis that mean of yield distribution in each time period is zero, and consider the mean parameter of yield distribution $\theta_{j}$. Third, change the hypothesis that variance in each time period is same, and consider the variance coefficient $\tau_{t}^{2}$. 
The joint distribution density of yield time series after changes is:

$$
p(r \mid \theta, \quad \tau, \kappa)=(2 \pi)^{-T / 2} \cdot \prod_{t=1}^{T} \tau_{t}^{-1} \cdot \prod_{j=0}^{N(\kappa)} \exp \left\{-\frac{1}{2} \sum_{t=\kappa_{j}+1}^{\kappa_{j+1}} \frac{\left(r_{t}-\theta_{j}\right)^{2}}{\tau_{t}^{2}}\right\} .
$$

Among them, $\theta=\left(\theta_{0}, \theta_{1}, \ldots, \theta_{N(\kappa)}\right)^{\prime}$ and $\tau=\left(\tau_{0}, \tau_{1}, \ldots, \tau_{T}\right)^{\prime}$. The interesting parameter is position of change point $\kappa$.

For the mean parameter, a prior setting easy for math processing with intuitive explanation is adopted here, $\theta_{j} \sim N\left(0, \delta_{j}^{-2}\right)$, and $j=0,1, \ldots, N(\kappa) ; \theta_{0}, \theta_{1}, \ldots, \theta_{N(\kappa)}$ are mutually independent. When $\delta_{j} \rightarrow+\infty$, it changes to the hypothesis of Inclan (1993) [21] that mean of yield is zero.

Since the joint distribution function of $\theta$ is:

$$
p(\theta)=(2 \pi)^{-\frac{N(\kappa)+1}{2}} \cdot \prod_{j=0}^{N(\kappa)} \delta_{j} \exp \left(-\frac{\theta_{j}^{2} \delta_{j}^{2}}{2}\right) .
$$

Using Bayesian formula to integral $\theta$

$$
\begin{aligned}
& p(r \mid k)=\int p(r \mid \theta, \tau, k) p(\theta \mid \delta) d \theta \\
& =\int_{-\infty}^{\infty} \int_{-\infty}^{\infty} \mathrm{L} \int_{-\infty}^{\infty}(2 \pi)^{-\frac{T+N(k)+1}{2}} \cdot \prod_{t=1}^{T} \tau^{-1} \cdot \prod_{j=0}^{N(k)} \delta_{j} \\
& \cdot \exp \left\{-\frac{1}{2} \sum_{t=k_{j}+1}^{k_{J+1}} r_{t} \tau_{t}^{-2}\right\} \exp \left\{-\frac{1}{2} \Delta_{j}\left[\theta_{j}^{2}-\frac{\theta_{j}}{\Delta_{j}} \sum_{t=k_{j}+1}^{k_{J+1}} 2 r_{t} \tau_{t}^{-2}\right]\right\} d \theta_{1} d \theta_{2} \mathrm{~L} d \theta_{N(k)} \\
& \Delta_{j}=\delta_{j}^{2}+\sum_{t=k_{j}+1}^{k_{J+1}} \tau_{t}^{-2} .
\end{aligned}
$$

Use the integral formula of multivariate normal distribution, namely:

$I F x_{i} \sim N\left(\mu_{i}, \sigma_{i}^{2}\right), i=1,2, \ldots, n$ THEN $\int_{-\infty}^{\infty} \int_{-\infty}^{\infty} \mathrm{K} \int_{-\infty}^{\infty} \exp \left\{-\frac{1}{2}\left[\sum_{i=1}^{n}\left(x_{i}-\mu_{i}\right)^{2} \sigma_{i}^{-2}\right]\right\} d x_{1} d x_{2} \mathrm{~K} d x_{n}=(2 \pi)^{\frac{n}{2}} \prod_{i=1}^{n} \sigma_{i}$.

The form of changing Formula (5) to Formula (6) is as follows:

$$
\begin{aligned}
p(r \mid k)=\int p(r \mid \theta, \tau, k) p(\theta \mid \delta) d \theta & (2 \pi)^{-\frac{T+N(k)+1}{2}} \cdot \prod_{t=1}^{T} \tau^{-1} \cdot \prod_{j=0}^{N(k)} \delta_{j} \exp \left\{-\frac{1}{2} \sum_{t=k_{j}+1}^{k_{j+1}} r_{t} \tau_{t}^{-2}+\frac{1}{8 \Delta_{j}}\left(\sum_{t=k_{j}+1}^{k_{j+1}} 2 r_{t} \tau_{t}^{-2}\right)^{2}\right\} \\
& \cdot \int_{-\infty}^{\infty} \int_{-\infty}^{\infty} \mathrm{L} \int_{-\infty}^{\infty} \exp \left\{-\frac{1}{2} \Delta_{j}\left[\theta_{j}^{2}-\frac{1}{2 \Delta_{j}} \sum_{t=k_{i}+1}^{k_{j+1}} 2 r_{t} \tau_{t}^{-2}\right]\right\} d \theta_{1} d \theta_{2} \mathrm{~L} d \theta_{N(k)}
\end{aligned}
$$

Formula (7) corresponds to reference formula (6) to eliminate $\theta$, thus to obtain

$$
p(r \mid k)=\int p(r \mid \theta, \tau, k) p(\theta \mid \delta) d \theta
$$

$$
=(2 \pi)^{\frac{T}{2}} \cdot \prod_{t=1}^{T} \tau^{-1} \cdot \prod_{j=0}^{N(k)} \frac{\delta_{j}}{\sqrt{\Delta_{j}}} \exp \left\{-\frac{1}{2} \sum_{t=k_{j}+1}^{k_{j+1}} r_{t}^{2} \tau_{t}^{-2}+\frac{1}{2 \Delta_{j}}\left(\sum_{t=k_{j}+1}^{k_{j+1}} r_{t} \tau_{t}^{-2}\right)^{2}\right\} .
$$

Then the processing method of Inclan(1993)[12] is further used. Assuming the probabilities of change points are mutually independent to obey Bernoulli distribution with the parameter as $\lambda$, the prior probability at position of change point is $p(\kappa)=\lambda^{N(\kappa)}(1-\lambda)^{T-N(\kappa)}$; therefore, the posterior probability density at position of change point is: 


$$
\begin{aligned}
& p(r \mid k) \propto p(r \mid k) p(k)= \\
& \left(\frac{\lambda}{1-\lambda}\right)^{N(k)}(2 \pi)^{\frac{T}{2}} \cdot \prod_{t=1}^{T} \tau^{-1} \cdot \prod_{j=0}^{N(k)} \frac{\delta_{j}}{\sqrt{\Delta_{j}}} \exp \left\{-\frac{1}{2} \sum_{t=k_{j}+1}^{k_{j+1}} r_{t}^{2} \tau_{t}^{-2}+\frac{1}{2 \Delta_{j}}\left(\sum_{t=k_{j}+1}^{k_{j+1}} r_{t} \tau_{t}^{-2}\right)^{2}\right\} .
\end{aligned}
$$

Obviously, when Formula (9) for posterior probability is maximal, the position of change point $\bar{\kappa}_{B}$ is the Bayesian Estimation of mean change point of yield time series.

\section{Model Solving}

The calculation amount of Formula (9) for solving the posterior probability is large, and the posterior probability ratio can be used to greatly simplify the calculation as follow: Assume several change points have been found, the vector at the position of change point is $\kappa$, and then continue to seek the next change point. Set the position of new change point to $h$, between certain adjacent change points, namely, $\kappa_{j}<h<\kappa_{j+1}$, constituting the vector $\kappa^{*}$ at the position of change point. Comparing two posterior probability densities, if the old probability density $p\left(\kappa^{*} \mid r\right)$ is larger than the new probability density $p\left(\kappa^{*} \mid r\right)$, it shows that the new change point does not exist. On the contrary, it shows that a new change point exists at the position $h$. Therefore, the change point can be detected through calculating whether the posterior probability ratio is larger than 1 .

Specific solution process is similar to ICSS algorithm, which can calculate the posterior probability ratio of the entire sample walkthrough $\mathrm{T}-1$ sample points starting from no change point through the MATLAB program, and then find the maximum sample of posterior probability ratio. If the maximum posterior probability ratio is larger than 1, this point is the first change point; otherwise, it shows that posterior probability supports no change point, and the algorithm is terminated. The first change point divides the entire time series into two sections, to calculate posterior probability ration of logarithm in two sections of samples, and find the maximum sample point of posterior probability ratio walkthrough T-2 sample points in total. If the maximum posterior probability ratio is larger than 1 , this point is the second change point; otherwise, it shows that posterior probability does not support two change points, and the algorithm is terminated. Therefore, repeat the above step until the maximum posterior probability ratio no larger than 1 .

As mentioned above, based on Formula (9), after the new change point $h$ is added, posterior probability ratios of the new solution and the previous solution is:

$$
\frac{p\left(\kappa^{*} \mid r\right)}{p(\kappa \mid r)}=\frac{\lambda}{1-\lambda} \cdot \frac{\delta_{1} \delta_{2}}{\delta} \cdot\left[\frac{\Delta}{\Delta_{1} \Delta_{2}}\right]^{1 / 2} \cdot \exp \left\{\frac{1}{2}\left[-R_{1}-R_{2}+R+\frac{T_{1}^{2}}{\Delta_{1}}+\frac{T_{2}^{2}}{\Delta_{2}}-\frac{T^{2}}{\Delta}\right]\right\} .
$$

Among them,

$$
R=\sum_{t=\kappa_{j}+1}^{\kappa_{j+1}} r_{t}^{2} \tau_{t}^{-2}, R_{1}=\sum_{t=\kappa_{j}+1}^{h} r_{t}^{2} \tau_{t}^{-2}, R_{2}=\sum_{t=h+1}^{\kappa_{j+1}} r_{t}^{2} \tau_{t}^{-2}, T=\sum_{t=\kappa_{j}+1}^{\kappa_{j+1}} r_{t} \tau_{t}^{-2}, T_{1}=\sum_{t=\kappa_{j}+1}^{h} r_{t} \tau_{t}^{-2}, T_{2}=\sum_{t=h+1}^{\kappa_{j+1}} r_{t} \tau_{t}^{-2}
$$

Here, $\delta$ is the mean parameter between the change point $\kappa_{j}$ and change point $\kappa_{j+1} ; \delta_{1}, \delta_{2}$ respectively indicate the mean parameter between $\kappa_{j}$ and $h$, and between $h$ and $\kappa_{j+1}$. It shows from Formula (10) that the calculation of posterior probability ratio is only limited to samples between adjacent change points, not involving samples of the entire time series. This is the basis of posterior probability ratio for large simplification of the calculation.

For setting of variance parameter $\tau_{t}^{2}$, referring to the definition of variance in statistics, the square of difference between time series value $r_{t}$ and mean $\theta_{j}$ is taken as the value of variance. Since the mean $\theta_{j}$ is unknown, the sample mean $\bar{\theta}_{j}$ is used. Namely, $\tau_{t}=\left|r_{t}-\bar{\theta}_{j}\right|, \kappa_{\mathrm{j}}<t \leq \kappa_{j+1}, j=0,1, \ldots, N(\kappa)$. 
Then setting two hyper-parameters: $\lambda, \delta$. The prior probability $\lambda$ of change point is first considered, namely, the probability whether each time series point is change point. The probability of one time series point as change point is assumed same as that of non-change point, namely, $\lambda=0.5$. Then, the variance coefficient $\delta$ of mean parameter is considered. As mentioned above, mean parameter is $\theta_{j} \sim N\left(0, \delta_{j}^{-2}\right)$, and $j=0,1, \ldots, N(\kappa)$. Under the condition that $\theta_{j}$ is known, the maximum likelihood estimate of variance coefficient is $\hat{\delta}_{j}=\left|\theta_{j}\right|^{-1}$. In fact, the mean $\theta_{j}$ is unknown; therefore, the sample mean $\bar{\theta}_{j}$ is used instead, namely, $\hat{\delta}_{j}=\left|\bar{\theta}_{j}\right|^{-1}$.

\section{Empirical Analysis}

In empirical research, we select Shanghai Composite Index (000001), because Shanghai Composite Index can almost completely reflect the performance of the entire market. Day closing data for 12 years from 2003 to 2014 are selected, and the monthly average closing index is calculated by month. According to Formula $r_{t}=\ln \left(P_{t} / P_{t-1}\right)$, the price data are converted to yield time series of logarithm. If put all their change points in price charts, the change points are shown in Figure 1 as vertical lines.

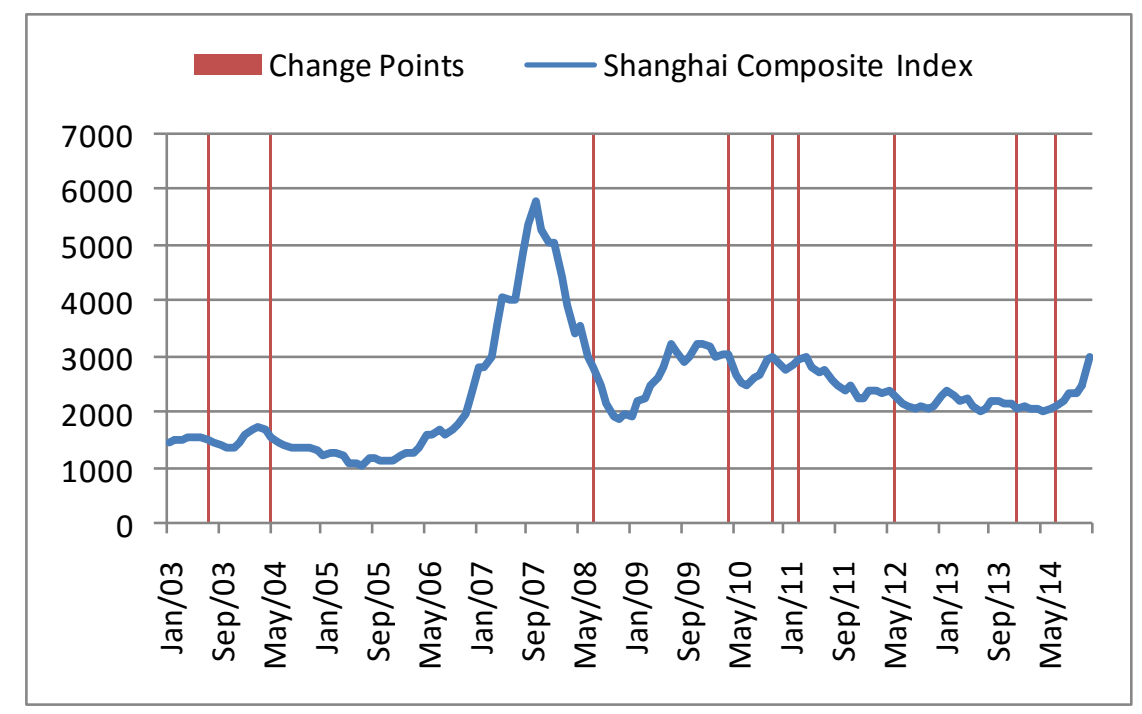

Fig. 1 Change points of Shanghai Composite Index

Based on the research of the Bayesian Method, we carries out two important improvements in the detection of structural changes in stock market:1) The assumed value is not used for variance parameter, but the real accumulated value similar to ICSS algorithm is used, to focus on the detection of mean change point of the yield. 2) Based on posterior probability ratio, an efficient solution algorithm similar to ICSS algorithm is put forward, to greatly overcome the disadvantage of large calculation amount of the Bayesian Method.

\section{Acknowledgment}

This research was supported by National Natural Science Foundation of China (No.71171128).

\section{References}

[1] Abraham B, Box G, Bayesian analysis of some outlier problems in time series, J. Biometrika. $1979,(66): 229-236$.

[2] Inclan, Detection of Multiple Changes of Variance Using Posterior Odds, J. Journal of Business\& Economic Statistics. 1993, (11):289-300. 